\title{
Automatic quantitative computed tomography measurement of longitudinal lung volume loss in interstitial lung diseases
}

\author{
Salim A. Si-Mohamed ${ }^{1,2} \cdot$ Mouhamad Nasser $^{3} \cdot$ Marion Colevray $^{4} \cdot$ Olivier Nempont $^{5} \cdot$ Pierre-Jean Lartaud $^{2}$. \\ Anna Vlachomitrou ${ }^{5}$. Thomas Broussaud ${ }^{2} \cdot$ Kais Ahmad $^{3}$. Julie Traclet ${ }^{3} \cdot$ Vincent Cottin $^{3} \cdot$ Loic Boussel $^{2,4}$
}

Received: 26 July 2021 / Revised: 9 November 2021 / Accepted: 24 November 2021 / Published online: 14 January 2022

(c) The Author(s) 2021

\begin{abstract}
Objectives To compare the lung CT volume (CTvol) and pulmonary function tests in an interstitial lung disease (ILD) population. Then to evaluate the CTvol loss between idiopathic pulmonary fibrosis (IPF) and non-IPF and explore a prognostic value of annual CTvol loss in IPF.

Methods We conducted in an expert center a retrospective study between 2005 and 2018 on consecutive patients with ILD. CTvol was measured automatically using commercial software based on a deep learning algorithm. In the first group, Spearman correlation coefficients ( $r$ ) between forced vital capacity (FVC), total lung capacity (TLC), and CTvol were calculated. In a second group, annual CTvol loss was calculated using linear regression analysis and compared with the Mann-Whitney test. In a last group of IPF patients, annual CTvol loss was calculated between baseline and 1-year CTs for investigating with the Youden index a prognostic value of major adverse event at 3 years. Univariate and log-rank tests were calculated. Results In total, 560 patients (4610 CTs) were analyzed. For $1171 \mathrm{CTs}$, CTvol was correlated with FVC ( $r$ : 0.86) and TLC $(r: 0.84)(p<0.0001)$. In 408 patients $(3332 \mathrm{CT})$, median annual CTvol loss was $155.7 \mathrm{~mL}$ in IPF versus $50.7 \mathrm{~mL}$ in nonIPF $(p<0.0001)$ over 5.03 years. In 73 IPF patients, a relative annual CT vol loss of $7.9 \%$ was associated with major adverse events (log-rank, $p<0.0001)$ in univariate analysis $(p<0.001)$.

Conclusions Automated lung CT volume may be an alternative or a complementary biomarker to pulmonary function tests for the assessment of lung volume loss in ILD.

Key Points

- There is a good correlation between lung CT volume and forced vital capacity, as well as for with total lung capacity measurements ( $r$ of 0.86 and 0.84 respectively, $p<0.0001$ ).

- Median annual CT volume loss is significantly higher in patients with idiopathic pulmonary fibrosis than in patients with other fibrotic interstitial lung diseases (155.7 versus $50.7 \mathrm{~mL}, p<0.0001$ ).

- In idiopathic pulmonary fibrosis, a relative annual CT volume loss higher than $9.4 \%$ is associated with a significantly reduced mean survival time at 2.0 years versus 2.8 years (log-rank, $p<0.0001$ ).
\end{abstract}

Keywords Tomography $\cdot$ Lung disease, interstitial $\cdot$ Pulmonary fibrosis $\cdot$ Idiopathic pulmonary fibrosis $\cdot$ Deep learning

Mouhamad Nasser and Marion Colevray contributed equally to this work.

Marion Colevray is a co-second author.

Salim A. Si-Mohamed

salim.si-mohamed@chu-lyon.fr

1 Radiology Department, Department of Cardiovascular and Thoracic Radiology, CHU Cardiologique Louis Pradel, Louis Pradel Hospital, 59 Boulevard Pinel, 69500 Bron, France

2 University of Lyon, University Claude-Bernard Lyon 1, UJM-Saint-Étienne, CNRS, Inserm, CREATIS UMR 5220, U1206, 69621 Lyon, France
3 National Reference Center for Rare Pulmonary Diseases, Louis Pradel Hospital, Hospices Civils de Lyon, UMR 754, INRAE, Claude Bernard University Lyon 1, Lyon, France

4 Radiology Department, Hôpital de La Croix-Rousse, 103 Grande rue de la Croix Rousse, 69004 Lyon, France

5 Philips Research, Suresnes, France 


\section{Abbreviations \\ CT Computed tomography \\ CTvol CT volume \\ FVC Forced vital capacity \\ HRCT High-resolution computed tomography \\ ILD Interstitial lung disease \\ IPF Idiopathic pulmonary fibrosis \\ MAE Major adverse event \\ PFT Pulmonary function test \\ TLC Total lung capacity}

\section{Introduction}

Interstitial lung diseases (ILDs) encompass a heterogeneous group of chronic and fibrotic lung diseases with distinct disease course and prognosis [1]. They may be associated with progressive lung volume loss with impaired quality of life, and in advanced stage, respiratory failure. Idiopathic pulmonary fibrosis (IPF), an inexorably progressive disease, is the most severe and lethal among others [2,3]. Survival is shortened in patients with lower forced vital capacity (FVC) at baseline and annual FVC decline $\geq 10 \%$. FVC has been thus proposed as a surrogate marker for disease progression and mortality in all ILDs and has been advocated as a primary outcome in major clinical trials in IPF [4-6]. However, FVC measurement is subject to inherent measurement variability and might be inaccurate in frail patients, advanced disease stages, and subjects with intractable cough $[7,8]$. Therefore, a new feasible, reproducible, and effortless surrogate biomarker is still needed.

Chest high-resolution computed tomography (HRCT) is mandatory for disease evaluation in patients with ILD and is used for diagnostic, monitoring, and prognostic purposes. The current guidelines recommend that pulmonary function tests (PFT) and chest HRCT are both fundamental in patient follow-up [9-11]. Moreover, CT loss volume derived from visual or automated quantification of the lung volumes on HRCT has shown great interest for assessing the degree of severity, disease progression, and mortality in IPF and systemic sclerosis-associated ILD, in a relatively small ILD population [12-15]. Yet, there is a dearth of data on the role of longitudinal CT lung volume loss and annual decline and its prognostic in the IPF population.

Therefore, using a newly commercially available deep learning algorithm for automatic quantification of lung CT volume, we compare the lung CT volume and pulmonary function tests in a large interstitial lung diseases population. Then, we evaluate the longitudinal CT volume loss between IPF and non-IPF populations and explore a predictive value of annual CT volume loss in the IPF population.

\section{Materials and methods}

\section{Study population}

We conducted a retrospective, observational, longitudinal study between February 2005 and July 2018 in an ILD expert center (Louis Pradel Hospital, Hospices Civils de Lyon, Lyon, France). Clinical, functional, and imaging data for consecutive patients that underwent at least one unenhanced HRCT study for fibrotic ILD were collected. The diagnosis of ILD was made in multidisciplinary discussion according to international guidelines at the time of the patient's presentation. Data usage policy of the "Hospices Civils de Lyon" in terms of confidentiality, anonymization, and security was applied for each study, and approval was obtained from our local committee. Institutional review board approval was obtained for the study, and patient consent was waived.

\section{CT studies}

For the comparison between the PFT and CT volume (CTvol), only patients having a CT study within the two weeks of the PFT were considered eligible. For the longitudinal CTvol loss evaluation within non-IPF and IPF groups, patients who underwent more than four $\mathrm{CT}$ examinations were included. Finally, for the predictive analysis of CT volume loss in IPF patients, patients who had a baseline CT and a 1-year follow-up CT $( \pm 10$ days) were included. For all CT examinations, patients with a history of recent (within 3 months) acute exacerbation, pneumothorax, pleural effusion, or lower respiratory tract infection and confirmed by two senior radiologists (with 6 and 20 years of experience in chest imaging, S.S-M. and L.B., respectively) were excluded in order to be representative of the chronic disease course of these fibrotic ILDs.

\section{CT protocols}

All HRCT acquisitions were performed at the end of deep inspiration. The data were collected retrospectively from all CT examinations on several systems over the years: GE Medical Systems (Revolution GSI), Philips (Brilliance 40, Brilliance 64, iCT 256, Ingenuity CT, IQon, Spectral CT), Siemens (Somatom Definition AS and AS +). The scanning parameters were as follows: tube voltage $=100-140 \mathrm{kVp}$ (mean \pm SD: $121 \pm 9 \mathrm{kVp}$ ), helical scan mode. The mean slice thickness was $1.8 \pm 0.8 \mathrm{~mm}$ (range: $0.9-3 \mathrm{~mm}$ ). 


\section{Automatic quantification of lung CTvol}

We used commercially available software implemented in a clinical workstation (CT Pulmo Auto Results, provided under a research contract; IntelliSpace Portal ISP11.1, Philips Healthcare). This software is a U-net-based deep learning algorithm and allows the lung segmentation with the exclusion of the main airways including the trachea, stem, lobar bronchi, and the main vessels. Lung CTvol was expressed in liters (L) (Fig. 1).

\section{Clinical respiratory functional test data}

Patients' data on demographics and PFTs were collected from patients' records. PFTs were performed in all patients according to ATS/ERS official statement [16]. Total lung capacity (TLC) was measured with body plethysmography, and forced vital capacity (FVC) by spirometry, both expressed in liters (L).

\section{Statistical analysis}

The data are expressed as mean \pm standard deviation (SD) and median with interquartile range (IQR) for continuous variables. Categorical variables were presented as frequency (percentage). Distributions were tested for normality using the D'Agostino-Pearson test. Variables were compared using two paired Student t-test or Wilcoxon rank-sum test, as appropriate. For comparison purposes, differences in lung CTvol and functional volumes were compared using the Wilcoxon matched-pairs signed-rank test. For comparison between CTvol and respiratory volumes (FVC and TLC), a
Bland-Altman analysis (bias, limits of agreement), a linear regression analysis (95\% confidence interval, $R^{2}$ ), and Spearman correlation coefficients and their $95 \%$ confidence were calculated.

For estimation of the daily and annual CTvol loss during follow-up, linear regression between all individual CT across time was calculated. The Mann-Whitney test was used to compare daily and annual CTvol loss between IPF and non-IPF groups.

For determining a predictive CTvol loss in the IPF population for major adverse events (MAE), i.e., death and transplantation, we first calculated the absolute annual and relative CTvol loss between CT baseline and 1-year follow-up $\mathrm{CT}$ of each patient that underwent a minimal 3-year followup or had died or undergone transplantation within 3 years. Then, a receiver operator characteristic (ROC) analysis was used to evaluate the greater baseline CTvol and CTvol loss values with the Youden index after having dichotomized the population with or without MAE at 4 years after the first CT study (baseline). Accordingly, to this value, a chi-square test with Yates' continuity correction was used to test the association between categorical variables (MAE at 4 years, baseline CTvol, annual and relative CTvol loss). Univariate Cox regressions were performed. A Kaplan-Meier statistical analysis was used to test the survival rate in the IPF population with the best predictor greater and lower than the threshold tested.

Statistical analysis was performed using the SPSS $®$ software $\mathrm{v} 23$ (IBM) and R software v3.5. A $p$ value less than 0.05 was considered significant. The Bonferroni correction was used to adjust the $p$ values in the longitudinal and predictive studies, i.e., less than 0.01 , respectively.

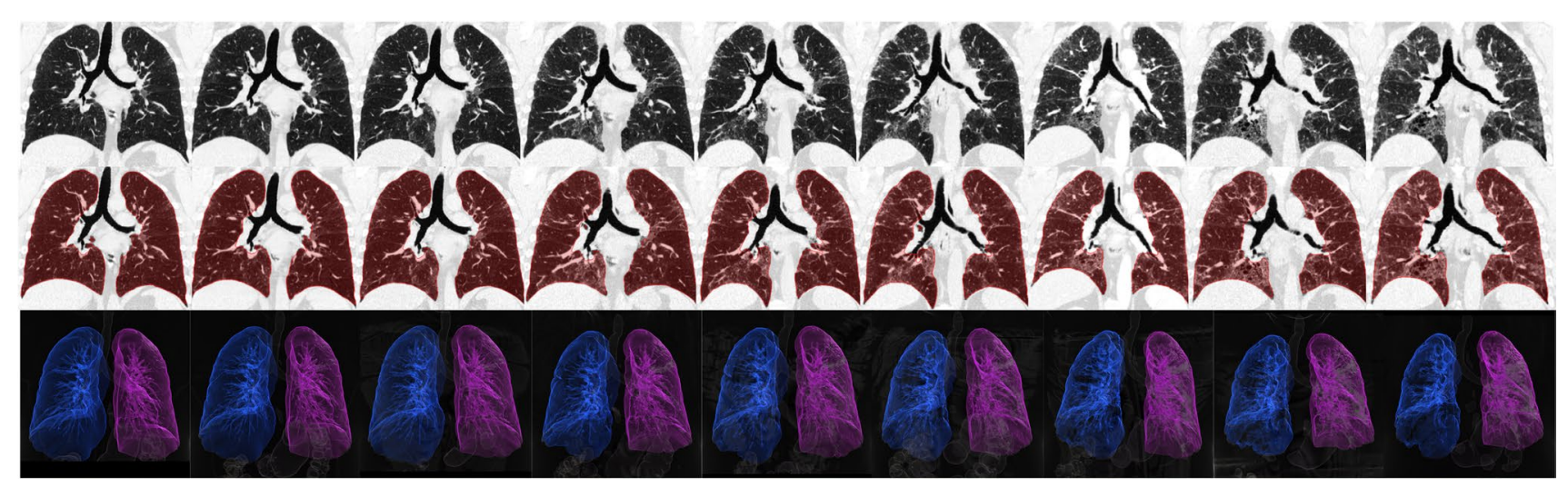

Fig. 1 Automated segmentation of 9 CT lung volumes over a 6-year follow-up in a 58 -year-old male affected by idiopathic pulmonary fibrosis. The first row represents the coronal images. The second row represents the mask segmentation of the lung. The third row repre- sents the 3D volume after lung segmentation using commercially available software based on a U-net-based deep learning algorithm (CT Pulmo Auto Results un-released, provided under a research contract; IntelliSpace Portal ISP11.1, Philips Healthcare) 


\section{Results}

\section{Study population}

In total, 560 patients were included in the study (341 men (61\%); mean age of $65.4 \pm 13.9$ years), corresponding to 4657 CT studies (mean CT studies per patient of $6.2 \pm 4.6$ ) (Fig. 2).

\section{Relation between CTvol and pulmonary function tests}

We analyzed 424 patients corresponding to $1171 \mathrm{CT}$ studies (Table 1). There was a good correlation between CTvol and FVC (Spearman coefficient of 0.86 (IC95\%: 0.84-0.87, $p<0.0001$ ) as well as between CTvol and TLC (Spearman coefficient of 0.84 (IC95\%: 0.82-0.86, $p<0.0001$ ) (Table 2). Bland-Altman analysis between CTvol and FVC revealed a positive proportional bias of 0.97 , with $95.0 \%$ limits of agreement from -0.27 to $2.21 \mathrm{~L}$ and a negative proportional bias between CTvol and TLC of -0.68 , with $95.0 \%$ limits of agreement from -2.05 to $0.69 \mathrm{~L}$ (Table 2 and Fig. 3).
Table 1 Population characteristics of the comparative study

\begin{tabular}{ll}
\hline Population number $(\boldsymbol{n})$ & 424 \\
\hline Total CT studies & 1171 \\
CT studies per patient, mean \pm SD & $2.8 \pm 1.8$ \\
Age (year-old), mean \pm SD & $64.6 \pm 13.3$ \\
Sex (male), $n(\%)$ & $256(60.4)$ \\
Interstitial lung disease type, $\boldsymbol{n}(\%)$ & \\
Idiopathic pulmonary fibrosis & $108(25.5)$ \\
Unclassifiable ILD & $74(17.4)$ \\
Systemic sclerosis & $64(15.1)$ \\
Combined pulmonary fibrosis and emphysema & $46(10.8)$ \\
Interstitial pneumonitis with auto-immune features & $37(8.7)$ \\
Dermatomyositis ILD & $25(5.9)$ \\
Hypersensitivity pneumonitis & $22(5.2)$ \\
Sjögren syndrome & $19(4.5)$ \\
Rheumatoid arthritis & $14(3.3)$ \\
Other (fibrotic COP, pneumoconiosis) & $11(2.6)$ \\
Sarcoidosis & $4(0.9)$ \\
\hline
\end{tabular}

Footnote. $n$ is the patient number, $C O P$ cryptogenic organizing pneumonia, ILD interstitial lung disease

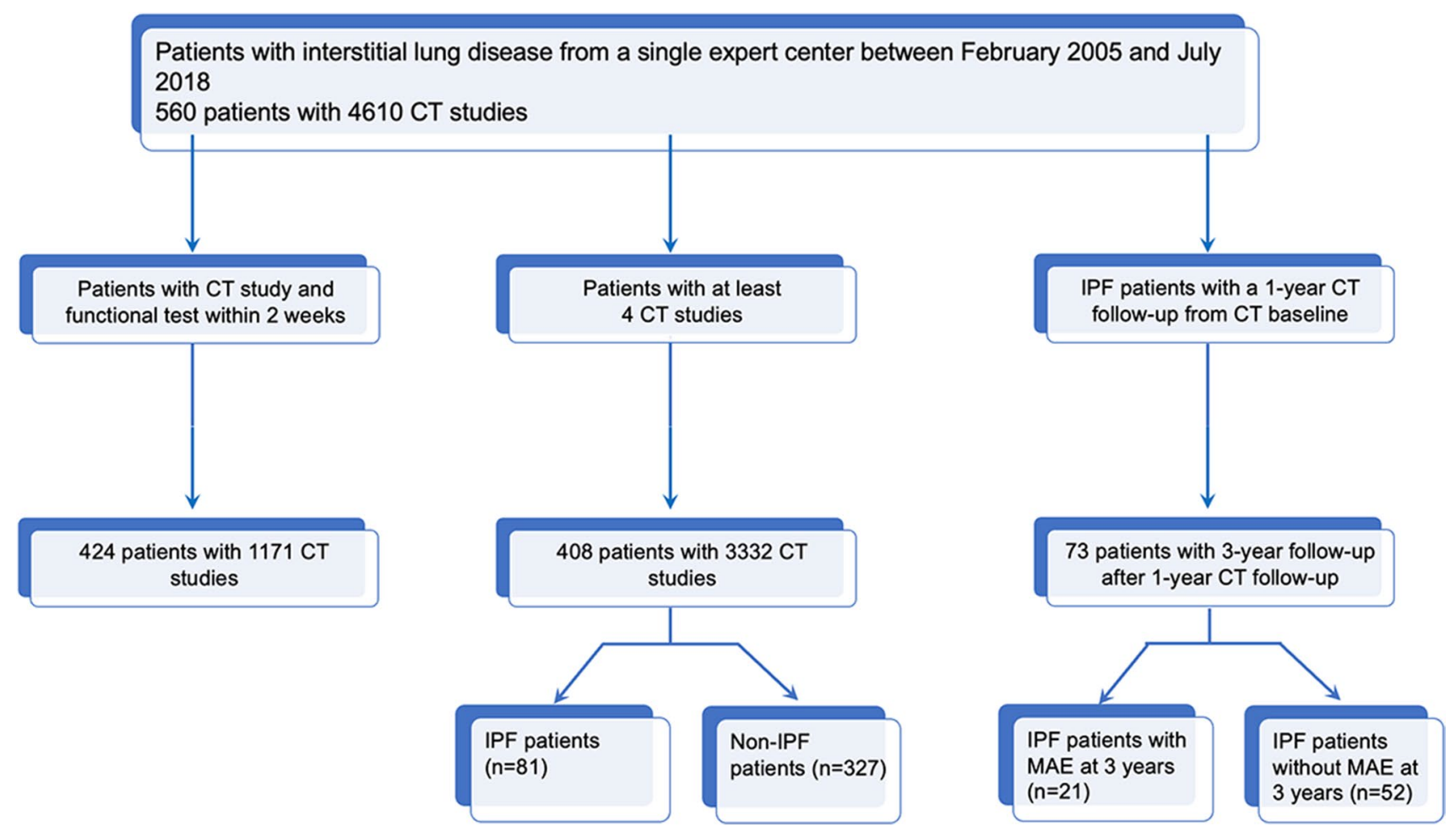

Fig. 2 Study flow chart. Three different yet partly overlapping groups of patients were consecutively selected from the population of 560 patients: 296 patients are common to the first two groups; 53 patients are common to the last two groups 
Table 2 Comparison between lung CT volume and pulmonary function tests (forced vital capacity and total lung capacity) in patients with fibrotic interstitial lug diseases (results of 1171 pairs of CT analyzed)

\begin{tabular}{|c|c|c|c|c|}
\hline Analysis & Parameters & CTvol (L) & Forced vital capacity (L) & Total lung capacity (L) \\
\hline & Median (IQR) & $3.3(2.6-4.3)$ & $2.4(1.8-3.1)$ & $4.0(3.1-5.0)$ \\
\hline \multirow[t]{3}{*}{ Linear regression statistics } & Slope & & 1.18 & 0.93 \\
\hline & Offset & & 0.52 & 0.90 \\
\hline & $\mathrm{R}^{2}$ & & 0.76 & 0.73 \\
\hline \multirow[t]{2}{*}{ Correlation statistics } & $\mathrm{r}$ & & 0.86 & 0.84 \\
\hline & $95 \% \mathrm{CI}$ & & $0.84-0.87$ & $0.82-0.86$ \\
\hline \multirow[t]{4}{*}{ Bland-Altman statistics } & r $(95 \% \mathrm{CI})$ & & $0.86(0.84-0.87)$ & $0.84(0.82-0.86)$ \\
\hline & Bias (SD) & & $0.97(0.63)$ & $-0.68(0.70)$ \\
\hline & $95 \%$ limits of agreement & & $-0.27 ;-2.21$ & $-2.05 ;-0.69$ \\
\hline & Slope $(95 \%$ CI $)$ & & $0.84(0.79-0.95)$ & $-0.3(-0.40$ to -0.19$)$ \\
\hline
\end{tabular}

Footnote. FVC forced vital capacity, TLC total lung capacity, SD standard deviation, CTvol CT volume, LOA limits of agreement
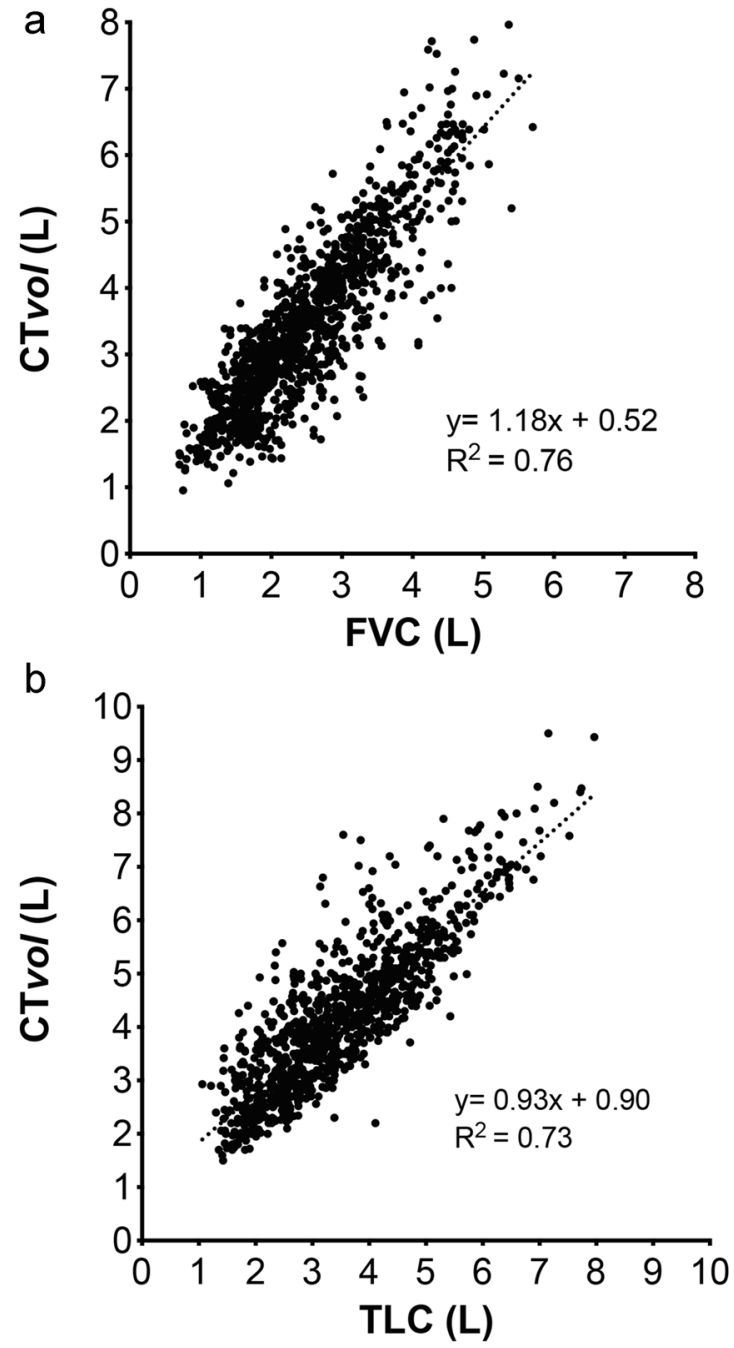
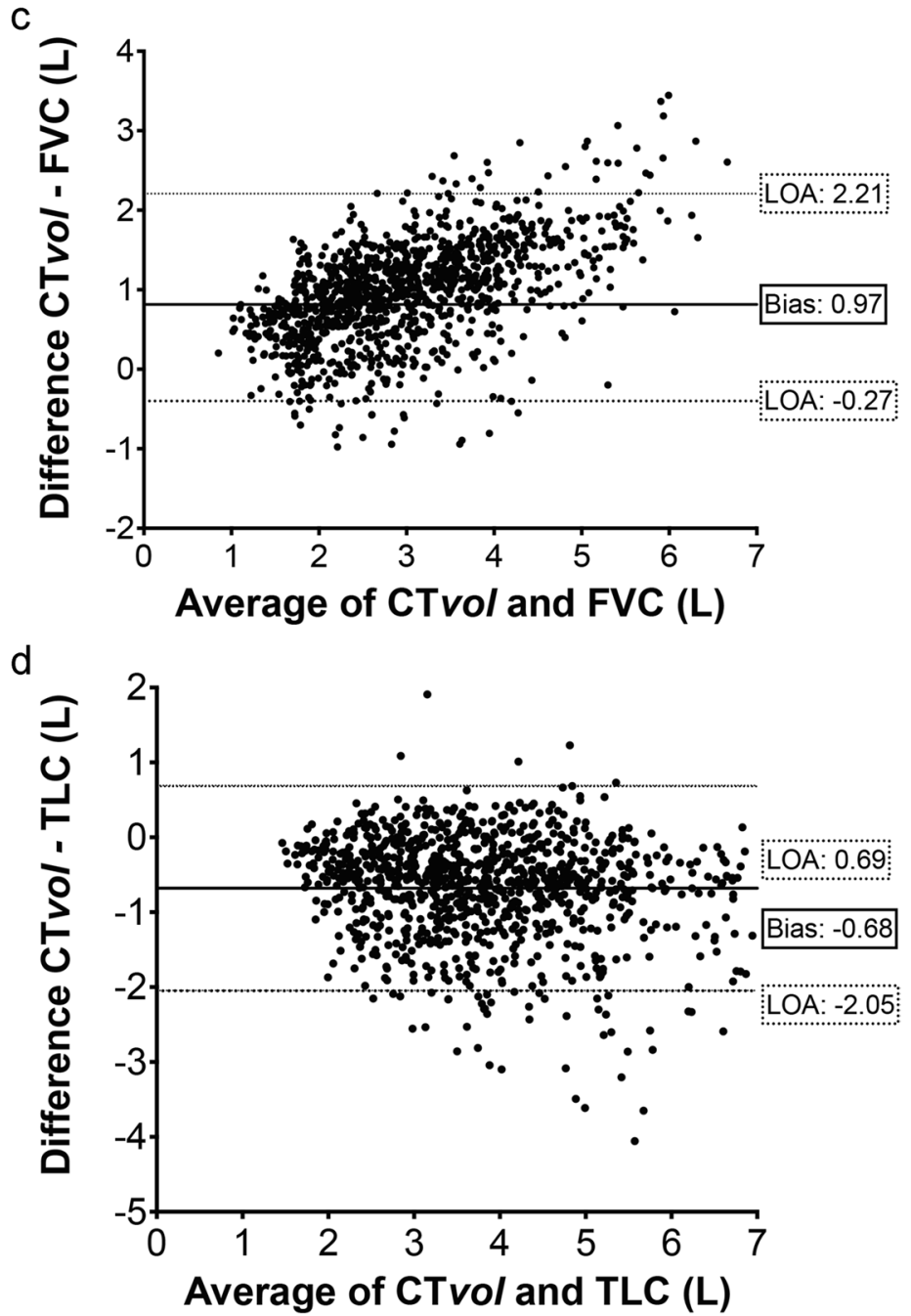

Fig. 3 Linear regression and Bland-Altman analysis graphs between CT volume (CTvol), forced vital capacity (a, b) and total lung capacity (c), d) 


\section{Longitudinal lung CTvol loss between IPF and non-IPF groups}

We analyzed 408 patients corresponding to 3332 CT studies, which were distributed in two groups: 81 (19.9\%) patients in the IPF group and $327(80.1 \%)$ in the non-IPF group (Table 3, Figs. 4 and 5). Median (IQR) follow-up was 1345 (1034-1867) days (approximately 3.7 years) in the IPF group and 2051 (1456-2881) days (approximately 5.7 years) in the non-IPF group. Patients with IPF had a mean \pm SD of $8.0 \pm 3.6 \mathrm{CT}$ studies while patients with nonIPF had a mean \pm SD of $8.2 \pm 3.7 \mathrm{CT}$ studies. Median (IQR) daily CTvol loss was $0.41 \mathrm{~mL}(0.05-0.80)$ in IPF group and $0.14 \mathrm{~mL}(-0.05-0.40)$ in the non-IPF group $(p<0.0001)$. Median (IQR) annual CTvol loss was $155.7 \mathrm{~mL}$ (49.9-304.5) versus $50.7 \mathrm{~mL}(-19.0-144.7)$, respectively $(p<0.0001)$ (Table 2).

\section{One-year lung volume loss from baseline in the IPF group}

Annual CTvol loss between the CT baseline and the 1-year CT follow-up of 73 patients with IPF were analyzed. Twenty-one MAEs during the 3-year follow-up period were reported with a mean \pm SD delay of $440 \pm 288$ days after the annual CT. Sixteen patients died with a mean \pm SD delay of $416 \pm 288$ days after the annual CT from pneumonia (6,
$38 \%)$, respiratory failure $(3,19 \%)$, acute exacerbation (4, $25 \%)$, lung cancer $(1,6 \%)$, sepsis $(1,6 \%)$, and pulmonary embolism $(1,6 \%)$. Five patients underwent single lung transplantation with a mean \pm SD delay of $542 \pm 197$ days after the annual CT. Eight patients were lost before the 3-year follow-up and were previously excluded from the initial population. Four patients underwent transplantation before the annual CT follow-up and were excluded from the predictive analysis. Dichotomized baseline characteristics of the study population by presence of MAE are summarized in Table 4 .

Based on a ROC statistical analysis, performed on baseline CTvol, absolute and relative annual CTvol loss, we found that a relative CTvol loss of $7.9 \%$ best matched for greater sensitivity and specificity, respectively of $81.0 \%$ and 69.2\%, with an AUC at 0.74 [0.60; 0.86]. Optimal threshold for absolute CTvol loss was $0.37 \mathrm{~L} /$ year $(76.2 \%$ sensitivity and $71.1 \%$ specificity, AUC $=0.73[0.60-0.84]$ ) and baseline CTvol was $3.19 \mathrm{~L}(57.1 \%$ sensitivity and $61.5 \%$ specificity, AUC $=0.56$ [0.41-0.71]). Using multiple univariate Cox regression models (Table 5), no difference was observed with respect to age, sex, and baseline CTvol. But we found a significant effect for annual absolute CTvol loss $(p<0.01)$ and relative annual CTvol loss $(p<0.001)$, both dichotomized, on patient survival. The categorical variable (death at 4 years) was significantly associated with the annual CTvol loss with a $p<0.001$ using a chi-square test with Yates' continuity correction. Log-rank test demonstrated a significant

Table 3 Population characteristics of the longitudinal study

\begin{tabular}{|c|c|c|c|c|}
\hline & IPF group $(n=81)$ & Non-IPF group $(n=327)$ & $\operatorname{ALL}(n=408)$ & $p$ \\
\hline CT studies & 627 & 2705 & 3332 & \\
\hline $\mathrm{CT}$ studies per patient, mean $\pm \mathrm{SD}$ & $8.0 \pm 3.6$ & $8.2 \pm 3.7$ & $8.2 \pm 3.6$ & \\
\hline Age (year-old), mean $\pm \mathrm{SD}$ & $68.3 \pm 0.6$ & $60.89 \pm 14.17$ & $62.4 \pm 13.7$ & $<0.0001$ \\
\hline Sex (male), $n(\%)$ & $67(82.7)$ & $167(50.7)$ & $233(57.1)$ & \\
\hline Follow-up (days), median (IQR) & $1345(1034-1867)$ & $2051(1456-2881)$ & $1819(1301-2697)$ & $<0.0001$ \\
\hline \multicolumn{5}{|l|}{ Interstitial lung disease type, $\boldsymbol{n}(\%)$} \\
\hline Idiopathic pulmonary fibrosis & & & $81(19.9)$ & \\
\hline Unclassifiable ILD & & & $69(16.9)$ & \\
\hline Systemic sclerosis & & & $72(17.6)$ & \\
\hline Combined pulmonary fibrosis and emphysema & & & $44(10.8)$ & \\
\hline Interstitial pneumonitis with auto-immune features & & & $35(8.6)$ & \\
\hline Dermatomyositis ILD & & & $31(7.6)$ & \\
\hline Hypersensitivity pneumonitis & & & $21(5.1)$ & \\
\hline Rheumatoid arthritis & & & $19(4.6)$ & \\
\hline Sjögren syndrome & & & $15(3.7)$ & \\
\hline Other (fibrotic COP, pneumoconiosis) & & & $15(3.7)$ & \\
\hline Sarcoidosis & & & $6(1.5)$ & \\
\hline Daily CTvol loss (mL), median (Q1-Q3)) & $0.41(0.05-0.80)$ & $0.14(-0.05-0.40)$ & $0.16(-0.01-0.46)$ & $<0.0001$ \\
\hline Annual CTvol loss (mL), median (Q1-Q3) & $155.7(49.9-304.5)$ & $50.7(-19.0-144.7)$ & $58.9(-5.5-167.8)$ & $<0.0001$ \\
\hline
\end{tabular}

Footnote. $\mathrm{n}$ is the patient number, COP: cryptogenic organizing pneumonia, ILD: interstitial lung disease. Values are expressed as median and $(\mathrm{IQR})$ or mean $\pm \mathrm{SD}$, as appropriate 
Fig. 4 Notched box-and-whiskers plot showing the annual CT Volume (CTvol) in IPF and non-IPF groups. The lower and upper margins of each box indicate the 25 th and 75 th percentile. Median is marked by the line in the box, and mean by the red dot. $95 \%$ confidence interval of the median is represented by the notches and outliers indicate the minimal and maximal values

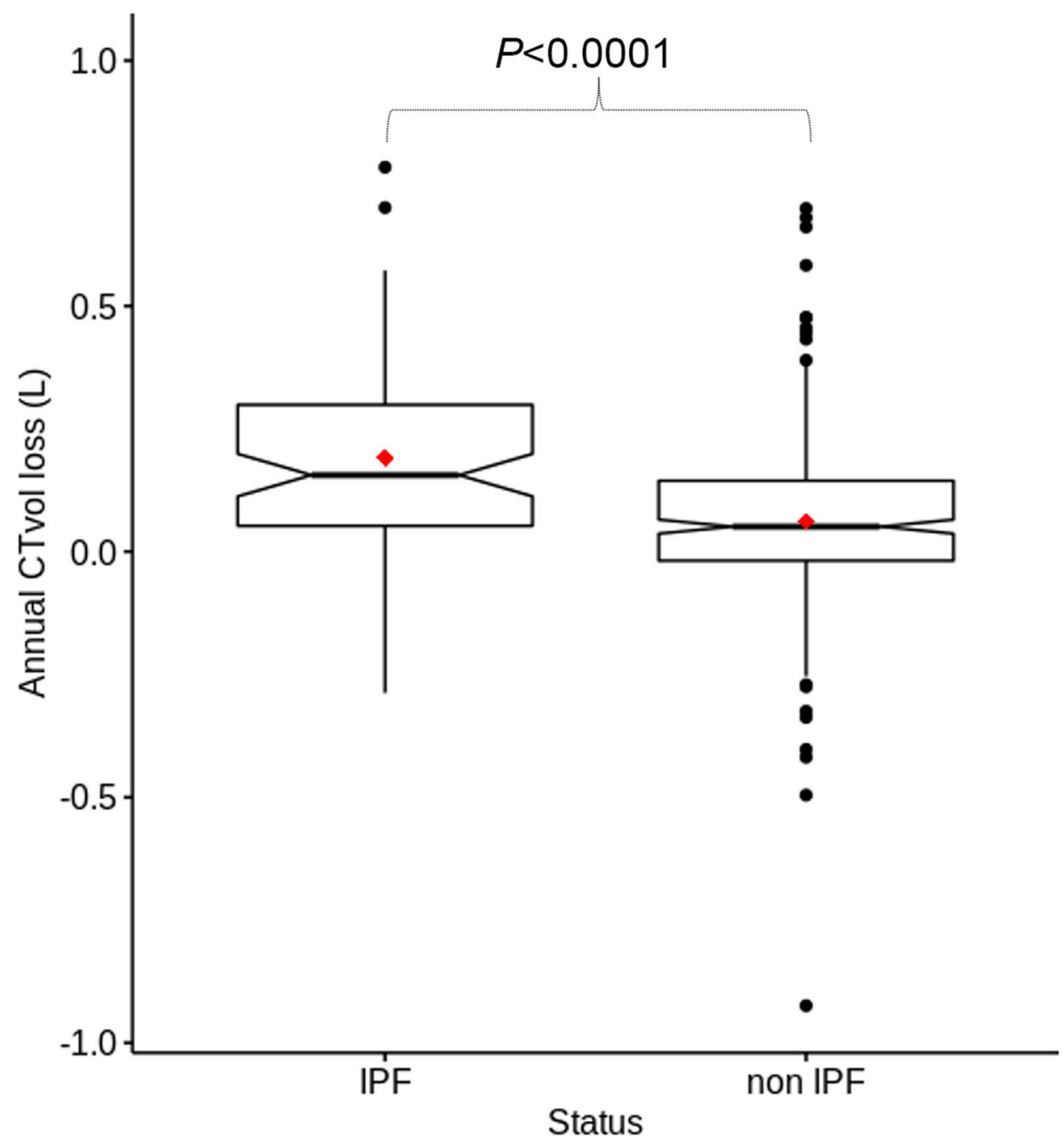

association as well with a $p<0.0001$. Kaplan-Meier survival curves are plotted in Fig. 6 according to $7.9 \%$ threshold for relative annual CTvol loss. Mean survival times were 1039 (SEM: 28) and 746 (SEM: 73) days respectively in the groups with lower and higher relative CTvol loss than $7.9 \%$.

\section{Discussion}

In the present study, we found that lung CT volume measurement, enabled by an automatic approach based on a deep learning algorithm, correlated strongly with FVC and TLC. Using longitudinal lung CT volume loss, we found that patients with IPF had a distinct disease course than other ILDs. In addition, in the IPF group, higher annual CT volume loss was associated with a worse prognosis.

The first results were the colinear relationship between CTvol with TLC, as previously shown [14, 17], but also with FVC among 1171 pairs of CT and PFT in a large cohort of ILD patients. CTvol was lower than TLC, such as previously demonstrated [18], which can be explained by patient positioning, e.g., the difference between CT exams (decubitus) and plethysmography (sitting position). Moreover, TLC measurement (body plethysmography) sums up all thoracic airspaces (including anatomic dead space) while CTvol only provides the measurement of parenchymal lung volume [19]. Thus, the delta between TLC and CTvol represents an anatomic dead space of $450 \mathrm{~mL}$. CTvol on the other hand, was higher than FVC, which can be explained by the contribution of the residual volume contrary to the evaluation of FVC. In addition, the Bland Altman analysis showed a positive proportional bias suggesting a higher difference between CTvol and FVC for high lung volumes.

From a clinical perspective, longitudinal assessment of volume loss may be more useful than a single measurement at baseline. The results of the present longitudinal study demonstrated a greater and faster CTvol loss among IPF than non-IPF patients, associated with shorter follow-up time (due to greater mortality) but a comparable number of CT exams. These findings are consistent with higher morbidity 
a

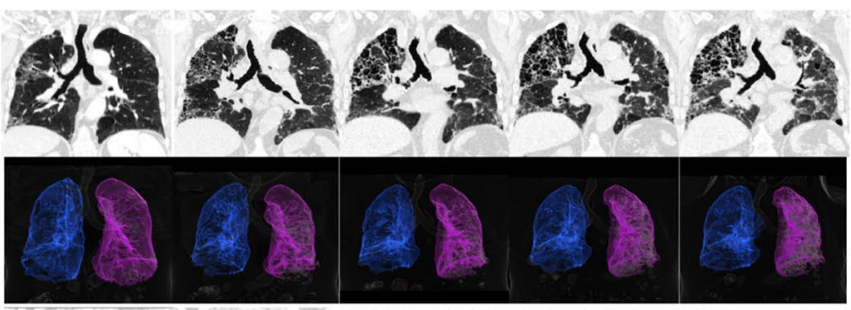

b
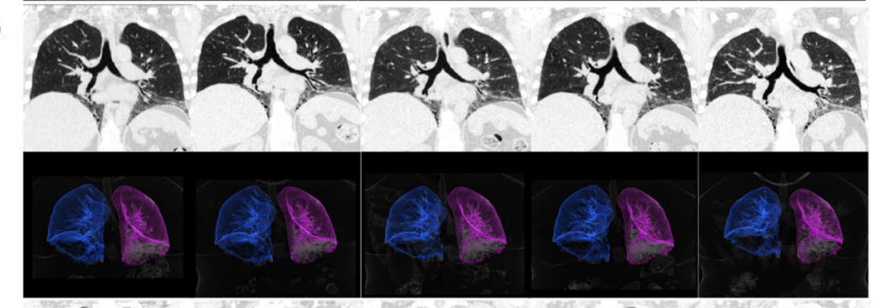

C
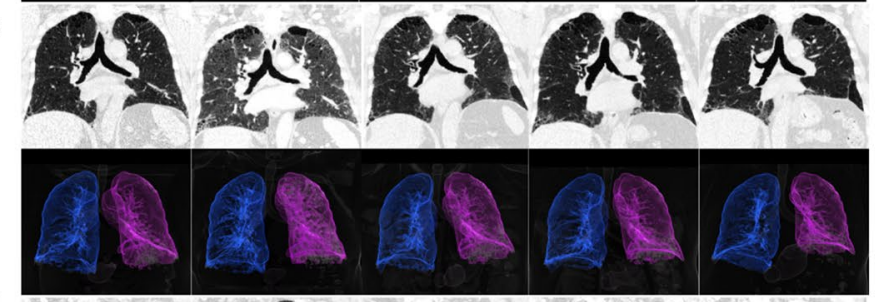

d

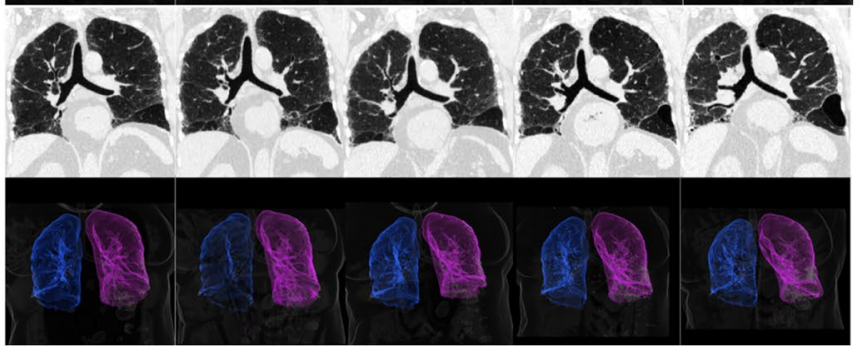

e

\section{Longitudinal CTvol loss}

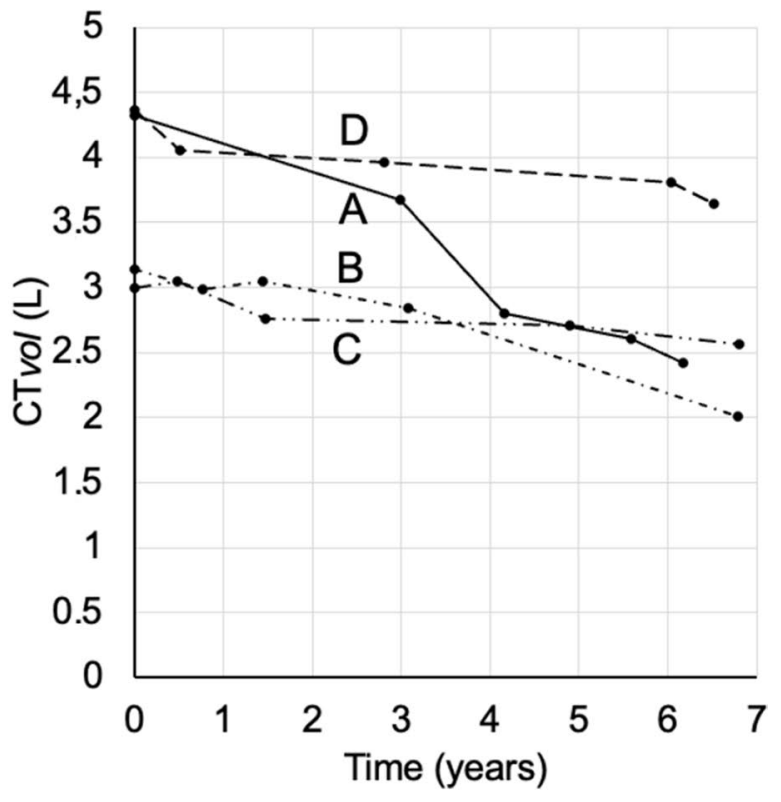

Fig. 5 Representative cases of interstitial lung diseases over 6-year follow-up. (a) Idiopathic pulmonary fibrosis, (b) systemic sclerosis, (c) unclassifiable interstitial lung disease, (d) combined pulmonary fibrosis and emphysema, (e) individual longitudinal CT volume loss graph

and mortality in IPF as compared to other fibrotic ILDs, leading to more frequent acute complications requiring more exams, and earlier death [20]. In addition, results from the longitudinal analysis showed in the IPF population a good agreement between annual CTvol loss and annual FVC loss as reported previously [21]. Of note, PFTs are time-consuming and require multiple measurements along with technical expertise in order to be reproducible [22-27].

Finally, in our attempt to clarify the interest for lung CTvol in the IPF population, we showed a significant association between the annual CTvol loss and the prognosis. We found a relative CTvol loss predictive value of $7.9 \%$ for death at 3 years after 1-year CT follow-up which is close to the $10 \%$ FVC decline threshold currently used as an endpoint, i.e., indicating disease progression in IPF clinical studies [28]. Using the $7.9 \%$ threshold, we found a mean survival time of 2.8 versus 2.0 years which is in line with the average 2.5-year survival time reported in IPF patients [29]. Interestingly, we found also an association between the absolute CTvol loss and mortality. In addition, the baseline CT volume was not associated with the prognosis contrary to the CT volume loss while there was a trend to a lower baseline $\mathrm{CT}$ in patients with poor prognosis. This result is supporting the findings of previous studies that highlighted the stronger prognostic value of the clinical and physiological parameters change compared to the baseline lung function in fibrotic ILD [30, 31]. However, the meaning of this finding may be limited due to the absence of normalization as a function of the age, sex, and height of the patient.

In this study, the strength was to use an automated solution based on a deep learning algorithm that allowed the analysis of a large cohort, previously limited by exclusive manual processing [21-23]. This solution is a commercially available fully automatic application that does not require any user interaction, besides installation, and that is part of the software suite available in the IntelliSpace Portal (Philips Healthcare). In our study, this was made available as a standalone to be deployed in a batch mode. But lung volumetric measurements can be done on-demand or in pre-processing mode as soon as the data from the scanner 
Table 4 Characteristics of patients with idiopathic pulmonary fibrosis

\begin{tabular}{|c|c|c|c|c|}
\hline & $\begin{array}{l}\text { No major adverse event at 3-year } \\
\text { follow-up }(N=52)\end{array}$ & $\begin{array}{l}\text { Major adverse event at 3-year } \\
\text { follow-up }(N=21)\end{array}$ & All $(n=73)$ & $p$ \\
\hline \multicolumn{4}{|l|}{ Age at baseline (years) } & \multirow[t]{4}{*}{$>0.05$} \\
\hline Mean (SD) & $71(7)$ & $66(13)$ & $69(9)$ & \\
\hline Median (Q1-Q3) & $70(68,76)$ & $70(58,73)$ & $70(65,76)$ & \\
\hline Min-Max & $50-82$ & $36-88$ & $36-88$ & \\
\hline \multicolumn{4}{|l|}{ Sex } & \multirow[t]{2}{*}{$>0.05$} \\
\hline Male & $43(82.7 \%)$ & $18(85.7 \%)$ & $61(83.6 \%)$ & \\
\hline \multicolumn{4}{|l|}{ Follow-up period (days) } & \multirow[t]{4}{*}{$<0.001$} \\
\hline Mean (SD) & $1095(0)$ & $440(336)$ & 907 (347) & \\
\hline Median (Q1-Q3) & $1095(1095,1095)$ & $517(115,721)$ & $1095(841,1095)$ & \\
\hline Min-max & $1095-1095$ & $12-1006$ & $12-1095$ & \\
\hline \multicolumn{4}{|l|}{ Baseline CTvol (L) } & \multirow[t]{4}{*}{$>0.05$} \\
\hline Mean (SD) & $3.425(1.051)$ & $3.147(0.898)$ & $3.345(1.011)$ & \\
\hline Median (Q1, Q3) & $3.316(2.658,3.906)$ & $3.131(2.614,3.678)$ & $3.304(2.614,3.896)$ & \\
\hline Min-max & $1.835-6.668$ & $1.480-4.977$ & $1.480-6.668$ & \\
\hline \multicolumn{4}{|c|}{ Absolute annual CTvol loss (L) } & \multirow[t]{4}{*}{0.003} \\
\hline Mean (SD) & $0.113(0.620)$ & $0.559(0.538)$ & $0.242(0.627)$ & \\
\hline Median (Q1-Q3) & $0.048(-0.242,0.455)$ & $0.488(0.367,0.772)$ & $0.275(-0.104,0.575)$ & \\
\hline Min-max & -1.171 to 2.096 & -0.670 to 1.657 & -1.171 to 2.096 & \\
\hline \multicolumn{4}{|c|}{ Relative annual CTvol loss (\%) } & \multirow[t]{4}{*}{0.001} \\
\hline Mean (SD) & $2.07(19.06)$ & $17.44(18.87)$ & $6.49(20.13)$ & \\
\hline Median (Q1-Q3) & $1.55(-7.21,13.93)$ & $16.87(9.29,28.05)$ & $6.32(-2.61,19.96)$ & \\
\hline Min-max & -52.14 to 50.10 & -33.40 to 51.16 & -52.14 to 51.16 & \\
\hline
\end{tabular}

Footnote: CTvol CT volume loss calculated between the baseline CT and the 1-year follow-up CT, SD standard deviation. Values are expressed as median and (IQR) or mean $\pm \mathrm{SD}$, as appropriate. Chi-square and Kruskal-Wallis tests performed on proportions or continuous variables respectively. $p$ values less than 0.01 were considered significant using the Bonferroni correction

Table 5 Univariate Cox regression models: CTvol parameters are dichotomized (*) after application of separate thresholds (baseline of $3.19 \mathrm{~L}$, decline of $0.37 \mathrm{~L}$ or $7.9 \%$ per year from baseline). Cox regression was significant for absolute and relative CTvol loss

\begin{tabular}{llcrr}
\hline & beta & HR (95\% CI & Wald.test & $p$ \\
\hline Age (years) & -0.049 & $0.95(0.91-0.99)$ & 5.1 & 0.02 \\
Sex (female vs male) & 0.14 & $1.2(0.34-3.9)$ & 0.05 & $>0.05$ \\
Baseline CTvol* & 0.52 & $1.7(0.72-4.0)$ & 1.4 & $>0.05$ \\
Annual CTvol loss* & 1.5 & $4.7(1.8-12)$ & 10 & 0.0014 \\
Relative annual CTvol loss* & 1.9 & $6.8(2.3-20)$ & 12 & 0.0006 \\
\hline
\end{tabular}

$p$ values less than 0.01 were considered significant using the Bonferroni correction

is available. Running time (including additional measurements and reporting) varies, depending on the IT network and system workload, typically less than 2-3 min. Analysis of the inter-observer variability between a manual and automatic segmentation reported by the vendor showed a median and interquartile range (IQR) of the absolute volume differences of 19.6 ml and 9.1-32.0 mL IQR (Q1-Q3) [32], which appears as a relatively small range in comparison to the CTvol loss values reported in our study. Taken together, this explains the feasibility for investigating lung volumes at $\mathrm{CT}$ in a large representative cohort of consecutive patients with fibrotic ILD in a center of expertise. In addition, this automatic approach may be of great interest in many cases where measuring PFTs can be challenging. PFTs, although generally reproducible, are also person-dependent (i.e., on the patient and on the technician or physician performing the test). Measuring lung volumes using a different method could help interpret the findings, as do complementary methods in other areas. One additional result from the CT that cannot be obtained by PFTs is the ability to measure lobar volumes that may help to define the clinical evolution in some fibrotic ILDs, as suggested recently 
Fig. 6 Three-year survival Kaplan-Meier curve and logrank test, as a function of a $7.9 \%$ threshold for the relative CTvol loss in the idiopathic pulmonary fibrosis population. Mean survival times are 1039 (SEM: 28) and 746 (SEM: 73) days respectively ( 2.8 versus 2.0 years)

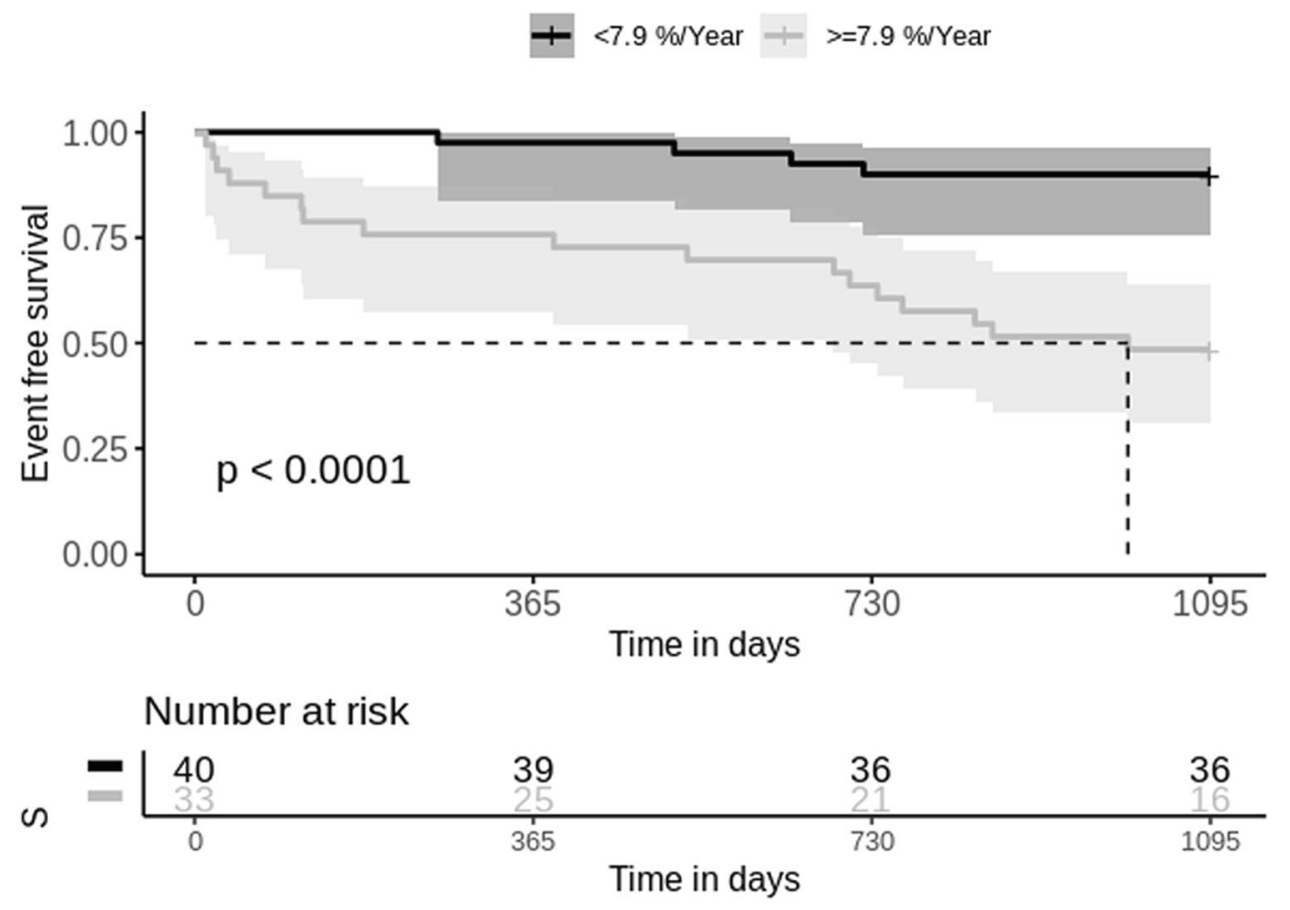

in pleuroparenchymal fibroelastosis [33]. Hence, our study represents a first step towards the identification of a new biomarker predicting physiological outcomes in order to consider the design of a new IPF mortality-risk score [34]. By taking into account additional CT parameters, automatic quantitative $\mathrm{CT}$ analysis could become a valid alternative or a complementary tool to pulmonary function tests in patients with ILD. However, the implementation of this technique in clinical practice warrants further prospective and controlled studies.

This study besides its retrospective and monocentric design has several limitations. First, CTvol measurement may vary with the individual's degree of inspiration during the CT examination. However, previous study reported an acceptable variability $(<10 \%)$ and a good repeatability of CTvol in patients with restrictive lung diseases, explained by reduced pulmonary compliance leading to less variable inspiratory volumes [36]. Hence, we assumed that inspiratory CT would be sufficient for patient follow-up although combined inspiratory and expiratory CT exams are often recommended [37]. Second, the presence of concomitant emphysema in ILD can underestimate disease progression despite FVC and TLC remaining stable [7], that is why estimation of lung volume solely with PFT can therefore sometimes be insufficient to monitor restrictive physiology. Nevertheless, our study did not take into account patients with concomitant emphysema. Hence, it would be interesting to perform additional lobar segmentation since pulmonary fibrosis takes place mainly at the basal lobes while emphysematous changes preferentially affect upper lobes [38]. Third, in the longitudinal study, patients with at least 4 CT studies were included to guarantee the quality of linear regressions which may be variable depending on the sampling pattern across time, i.e., may lead to influential points. However, in this real-life study, the CTs scans were not performed at fixed intervals, which can represent a bias. Fourth, our comparative study between the PFT results and CT volume values did not take into account the PFT values expressed as a percentage of the predicted value. Nevertheless, our aim was to evaluate the absolute precision of the CT for volume quantification. Fifth, the annual CT volume loss was assessed only in the IPF patients. Currently, there is no recommendation for performing an annual CT follow-up in the non-IPF patients, contrary to the IPF patients who undergo annual follow-up CT in our expert center according to the French recommendations for the management of IPF [39]. This explains the difficulty to assess the annual CT lung volume loss in non-IPF patients. In addition, non-IPF patients who had a follow-up CT probably had a CT due to a clinical indication including the occurrence of acute exacerbations, which may bias our analysis of the chronic disease course of the fibrotic ILD. Finally, cases were enrolled over a period of 13 years, and several different CTs were used; this in fact demonstrates that our approach can be implemented to various scenarios in a real-life setting.

In conclusion, automatic evaluation of the lung CT volume, in patients with ILD and particularly with IPF, may be an alternative or a complementary biomarker to pulmonary function tests for assessment of lung volume loss, in clinical care as in randomized trials. 
Funding The authors state that this work has not received any funding.

\section{Declarations}

Guarantor The scientific guarantor of this publication is Pr. Loic Boussel.

Conflict of interest Olivier Nempont and Anna Vlachomitrou are employees of Philips Healthcare from which the different kinds of software used in the study were designed. However, they neither had access nor control over the phantom data acquisition and analysis.

Statistics and biometry No complex statistical methods were necessary for this paper.

\section{Informed consent Not applicable.}

Ethical approval Data usage policy of the "Hospices Civils de Lyon" in terms of confidentiality, anonymization, and security was applied for each study, and approval was obtained from our local committee. Institutional review board approval was obtained for the study, and patient consent was not required.

\section{Methodology}

- Observational study

Open Access This article is licensed under a Creative Commons Attribution 4.0 International License, which permits use, sharing, adaptation, distribution and reproduction in any medium or format, as long as you give appropriate credit to the original author(s) and the source, provide a link to the Creative Commons licence, and indicate if changes were made. The images or other third party material in this article are included in the article's Creative Commons licence, unless indicated otherwise in a credit line to the material. If material is not included in the article's Creative Commons licence and your intended use is not permitted by statutory regulation or exceeds the permitted use, you will need to obtain permission directly from the copyright holder. To view a copy of this licence, visit http://creativecommons.org/licenses/by/4.0/.

\section{References}

1. Wijsenbeek M, Cottin V (2020) Spectrum of fibrotic lung diseases. N Engl J Med 383(10):958-968

2. Travis WD, Costabel U, Hansell DM et al (2013) An official American Thoracic Society/European Respiratory Society statement: update of the international multidisciplinary classification of the idiopathic interstitial pneumonias. Am J Respir Crit Care Med 188(6):733-748

3. Richeldi L, Collard HR, Jones MG (2017) Idiopathic pulmonary fibrosis. Lancet 389(10082):1941-1952

4. Noble PW, Albera C, Bradford WZ et al (2011) Pirfenidone in patients with idiopathic pulmonary fibrosis (CAPACITY): two randomised trials. Lancet 377(9779):1760-1769

5. King TE, Bradford WZ, Castro-Bernardini S et al (2014) A phase 3 trial of pirfenidone in patients with idiopathic pulmonary fibrosis. N Engl J Med 370(22):2083-2092

6. Richeldi L, du Bois RM, Raghu G et al (2014) Efficacy and safety of nintedanib in idiopathic pulmonary fibrosis. N Engl J Med 370(22):2071-82

7. Cottin V, Hansell DM, Sverzellati N et al (2017) Effect of emphysema extent on serial lung function in patients with idiopathic pulmonary fibrosis. Am J Respir Crit Care Med 196(9):1162-1171
8. Behr J (2017) Disease progression in idiopathic pulmonary fibrosis. FVC is not enough. Am J Respir Crit Care Med 196(9):1094-5

9. Raghu G, Remy-Jardin M, Myers JL et al (2018) Diagnosis of idiopathic pulmonary fibrosis. An Official ATS/ERS/JRS/ALAT Clinical Practice Guideline. Am J Respir Crit Care Med 198(5):e44-68

10. Cottin V, Crestani B, Cadranel J et al (2017) French practical guidelines for the diagnosis and management of idiopathic pulmonary fibrosis - 2017 update Full-length version. Rev Mal Respir 34(8):900-968

11. Lynch DA, Sverzellati N, Travis WD et al (2018) Diagnostic criteria for idiopathic pulmonary fibrosis: a Fleischner Society White Paper. Lancet Respir Med 6(2):138-153

12. Best AC, Meng J, Lynch AM et al (2008) Idiopathic pulmonary fibrosis: physiologic tests, quantitative CT indexes, and CT visual scores as predictors of mortality. Radiology 246(3):935-940

13. Torrisi SE, Palmucci S, Stefano A et al (2018) Assessment of survival in patients with idiopathic pulmonary fibrosis using quantitative HRCT indexes. Multidiscip Respir Med 13(1):43

14. Robbie H, Wells AU, Jacob J et al (2019) Visual and automated CT measurements of lung volume loss in idiopathic pulmonary fibrosis. AJR Am J Roentgenol 213(2):318-324

15. Chassagnon G, Vakalopoulou M, Régent A et al (2021) Elastic Registration-driven deep learning for longitudinal assessment of systemic sclerosis interstitial lung disease at CT. Radiology 298(1):189-198

16. Pellegrino R, Viegi G, Brusasco V et al (2005) Interpretative strategies for lung function tests. Eur Respir J 26(5):948-968

17. Raghu G, Scholand MB, de Andrade J et al (2016) FG-3019 anticonnective tissue growth factor monoclonal antibody: results of an open-label clinical trial in idiopathic pulmonary fibrosis. Eur Respir J 47(5):1481-1491

18. Garfield JL, Marchetti N, Gaughan JP, et al (2012) Total lung capacity by plethysmography and high-resolution computed tomography in COPD. Int J Chron Obstruct Pulmon Dis 7:119-126. https://doi. org/10.2147/COPD.S26419

19. Coates AL, Peslin R, Rodenstein D, Stocks J (1997) Measurement of lung volumes by plethysmography. Eur Respir J 10(6):1415-1427

20. Kolb M, Vašáková M (2019) The natural history of progressive fibrosing interstitial lung diseases. Respir Res 20(1):57

21. Ley B, Collard HR, King TE (2011) Clinical course and prediction of survival in idiopathic pulmonary fibrosis. Am J Respir Crit Care Med 183(4):431-440

22 Graham BL, Steenbruggen I, Miller MR et al (2019) Standardization of Spirometry 2019 Update. An Official American Thoracic Society and European Respiratory Society Technical Statement. Am J Respir Crit Care Med 200(8):e70-88

23. Wanger J, Clausen JL, Coates A et al (2005) Standardisation of the measurement of lung volumes. Eur Respir J 26(3):511-522

24. Hudson M, Assayag D, Caron M et al (2013) Comparison of different measures of diffusing capacity for carbon monoxide (DLCO) in systemic sclerosis. Clin Rheumatol 32(10):1467-1474

25. Dressel H, Filser L, Fischer R et al (2008) Lung diffusing capacity for nitric oxide and carbon monoxide: dependence on breath-hold time. Chest 133(5):1149-54

26. Pérez-Padilla R, Regalado-Pineda J, Mendoza L et al (2003) Spirometric variability in a longitudinal study of school-age children. Chest 123(4):1090-1095

27 Künzli N, Ackermann-Liebrich U, Keller R, Perruchoud AP, Schindler C (1995) Variability of FVC and FEV1 due to technician, team, device and subject in an eight centre study: three quality control studies in SAPALDIA. Swiss Study on Air Pollution and Lung Disease in Adults. Eur Respir J 8(3):371-6

28. Flaherty KR, Wells AU, Cottin V et al (2019) Nintedanib in progressive fibrosing interstitial lung diseases. N Engl J Med 381(18):1718-1727 
29. Ley B, Collard HR, King TE (2011) Clinical course and prediction of survival in idiopathic pulmonary fibrosis. Am J Respir Crit Care Med 183(4):431-440

30. Collard HR, King TE, Bartelson BB, Vourlekis JS, Schwarz MI, Brown KK (2003) Changes in clinical and physiologic variables predict survival in idiopathic pulmonary fibrosis. Am J Respir Crit Care Med 168(5):538-542

31. Jegal Y, Kim DS, Shim TS et al (2005) Physiology is a stronger predictor of survival than pathology in fibrotic interstitial pneumonia. Am J Respir Crit Care Med 171(6):639-644

32. CT Pulmo Auto Results (2021) ISP 1.1., Instructions for Use manual, Philips Healtcare

33. Nasser M, Si-Mohamed S, Turquier S, Traclet J et al (2021) Nintedanib in idiopathic and secondary pleuroparenchymal fibroelastosis. Orphanet J Rare Dis 16(1):419

34. Putman RK, Gudmundsson G, Axelsson GT et al (2019) Imaging patterns are associated with interstitial lung abnormality progression and mortality. Am J Respir Crit Care Med 200(2):175-183
35. Ley B, Elicker BM, Hartman TE et al (2014) Idiopathic pulmonary fibrosis: CT and risk of death. Radiology 273(2):570-579

36 Shin JM, Kim TH, Haam S et al (2017) The repeatability of computed tomography lung volume measurements: comparisons in healthy subjects, patients with obstructive lung disease, and patients with restrictive lung disease. PLoS One 12(8):e0182849

37. Gruden JF (2016) CT in idiopathic pulmonary fibrosis: diagnosis and beyond. AJR Am J Roentgenol 206(3):495-507

38. Jankowich MD, Rounds SIS (2012) Combined pulmonary fibrosis and emphysema syndrome. Chest 141(1):222-231

39. Cottin V, Crestani B, Valeyre D et al (2014) Diagnosis and management of idiopathic pulmonary fibrosis: French practical guidelines. Eur Respir Rev 23(132):193-214

Publisher's note Springer Nature remains neutral with regard to jurisdictional claims in published maps and institutional affiliations. 